\title{
Asymmetric simple exclusion process with periodic boundary driving
}

\author{
Vladislav Popkov, ${ }^{1,2}$ Mario Salerno, ${ }^{1}$ and Gunter M. Schütz, ${ }^{2,3}$ \\ ${ }^{1}$ Dipartimento di Fisica “E.R. Caianiello,” and Consorzio Nazionale Interuniversitario per le Scienze Fisiche della Materia (CNISM), \\ Università di Salerno, Baronissi, Italy \\ ${ }^{2}$ Interdisziplinäres Zentrum fur Komplexe Systeme, Römerstrasse 164, D-53117 Bonn, Germany \\ ${ }^{3}$ Institut für Festkörperforschung, Forschungszentrum Jülich, D-52425 Jülich, Germany \\ (Received 17 March 2008; revised manuscript received 29 May 2008; published 23 July 2008)
}

\begin{abstract}
We consider the asymmetric simple exclusion process (ASEP) on a semi-infinite chain which is coupled at the end to a reservoir with a particle density that changes periodically in time. It is shown that the density profile assumes a time-periodic sawtoothlike shape. This shape does not depend on initial conditions and is found analytically in the hydrodynamic limit. In a finite system, the stationary state is shown to be governed by effective boundary densities and the extremal flux principle. Effective boundary densities are determined numerically via Monte Carlo simulations and compared with those given by mean-field approach and numerical integration of the hydrodynamic limit equation which is the Burgers equation. Our results extend straightforwardly beyond the ASEP to a wide class of driven diffusive systems with one conserved particle species.
\end{abstract}

DOI: 10.1103/PhysRevE.78.011122

PACS number(s): 05.70.Ln, 64.60.Ht, 02.50.Ga

\section{INTRODUCTION}

Systems of driven diffusing particles attract attention because, despite their relative simplicity, they embrace a whole range of critical phenomena far from thermal equilibrium [1-3]. One of the remarkable features of these systems is the appearance of phase transitions induced by spatial boundaries of an open system which exchanges particles with external reservoirs $[4,5]$. A classical model where this can be studied in great detail is the so-called asymmetric simple exclusion process (ASEP) with open boundaries. This model describes the single-file random motion of particles with hard core exclusion and drift inside a finite system at the ends of which particles can be extracted or injected with some rates. This model was first introduced for describing the kinetics of protein synthesis [6,7] and has since then been generalized in many ways for describing the motion of various kinds of molecular motors [8-10]. Due to its conceptual simplicity it also plays a fundamental role in traffic flow theory $[11,12]$ and many other settings where driven diffusion of interacting particles plays a role.

By now, the dynamics of the ASEP (as well as its stationary bulk behavior) is rather well-understood. For our purposes we note that the exact stationary distribution has been determined analytically $[13,14]$, while the coarse-grained dynamics of shocks and localized excitations in the evolution of the particle density can be understood using hydrodynamic limit equations $[15,16]$. The latter provides a full description of the evolution of the local density under Eulerian scaling. It has been shown rigorously [17] to be given by the famous Burgers equation used for the description of the dynamics of shocks in dissipative systems [18].

The vast body of knowledge about the ASEP has been obtained for time-homogeneous conditions where the boundary rates are kept constant in time. In contrast, very little is known when the environment of this open system changes nonadiabatically in time on scales that are comparable to the macroscopic Eulerian hydrodynamic regime. This has to be modeled by time-dependent boundary rates which, to our knowledge, has not yet been attempted for the ASEP with open boundaries. It is the purpose of this work to report simulation results for a natural time-periodic setting and to analyze these data in the framework of the hydrodynamic theory.

The paper is organized as follows. In Sec. II we define the model and present our simulation data. The data for the Eulerian low-frequency regime of a semi-infinite system are then analyzed and explained by extending hydrodynamic theory to incorporate time-dependent boundary conditions (Sec. III). This analysis allows us to predict the phase diagram of an open finite system with two boundaries. This prediction and its numerical verification is given in Sec. IV. We end with a summary of our results and some conclusions (Sec. V).

\section{ASEP MODEL ON A SEMILINE WITH TIME PERIODIC BOUNDARY}

We consider the ASEP defined on a semi-infinite chain $0\{k \in \mathbb{Z}, k \leq 0\}$ with a right boundary site $k=0$. Each site of the chain is either occupied by one particle or empty. We denote the local occupation number by $n_{k} \in\{0,1\}$. Particles attempt to jump to the right or to the left neighboring site after an exponentially distributed random time with parameter $p+q$, normalized as $p+q=1$. The rate at which a particle attempts to hop to the right (left) is $p(q)$. If the target site is empty, the attempt is successful and the particles moves. Otherwise, it does not jump (hardcore exclusion rule). At the boundary site $k=0$ a particle can be extracted with the rate $\beta$ (if the boundary site is occupied) or be injected into it (if the boundary site is empty) with rate $\delta$. We choose $\beta=p(1$ $\left.-\rho_{R}\right), \delta=q \rho_{R}$ so that the boundary may be thought of as being coupled to a reservoir of density $\rho_{R}$ [19]. We consider the boundary reservoir density $\rho_{R}$ to be a periodic function of time with frequency $\nu$, switching between the values $\rho_{R}=0$ and $\rho_{R}=1$ according to 


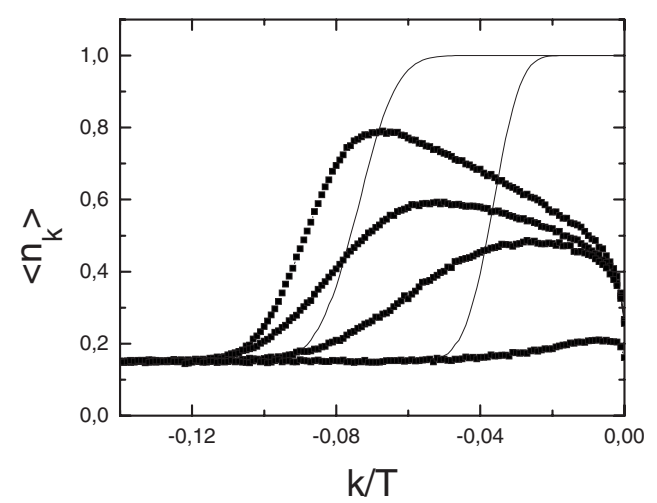

FIG. 1. Snapshots of density profiles averaged over $3 \times 10^{4}$ histories, during the red light $t=0.75 T, t=T$ (lines) and during the green light $t=1.1 T, 1.2 T, 1.3 T, 1.5 T$ (points). Parameters: $T=1000$. Initial state is a homogeneous one with the density $\left\langle n_{k}(0)\right\rangle=0.15$.

$$
\rho_{R}(t)=\frac{1}{2}\{1+\operatorname{sgn}[\sin (2 \pi \nu t)]\} .
$$

In traffic flow problems such a boundary condition models a traffic light with $\rho_{R}(t)=0$ during the "green light" halfperiods $t \subset\left[0, \frac{T}{2}\right],\left[T, \frac{3 T}{2}\right], \ldots$, and $\rho_{R}(t)=1$ during "red light" half-periods $\tau_{\text {red }} \subset\left[\frac{T}{2}, T\right],\left[\frac{3 T}{2}, 2 T\right], \ldots$, where $T=1 / \nu$. In analogy with this we refer to Eq. (1) as a traffic light boundary condition. For a study of such a switching in a related system, see [20].

To investigate the effects of this boundary condition on the dynamics of the ASEP on a semiline we have carried out Monte Carlo simulations of the model for various frequencies $\nu$. We have concentrated mainly on the case of a totally asymmetric simple exclusion process (TASEP), $q=0, p=1$, and focused our interest on stationary behavior in the sense that all macroscopic quantities behave periodically in time with period $T$. The initial state was prepared in an ensemble of particles randomly distributed with the density $\lambda$, which is stationary in the infinite system. In the presence of periodically varying boundary rates, rather complicated dynamics is observed. For small ingoing fluxes $j_{i n}=\lambda(1-\lambda)$, the system develops a jam at the boundary during the red light periods, which is dissolving completely during the green light periods, Fig. 1. However, if $\lambda$ (and consequently, the inflow flux) exceeds some critical value $\lambda>\lambda_{c}(\nu)$, the jam at the boundary is not dissolved completely, but starts to propagate inside the system. The amplitude of the shock front is not constant, it increases and drops during each red-green period, see Fig. 2. Consequently, shock front is not propagating steadily, but its velocity changes, and in particular it may advance and retract during each red-green period. The net shock advance after a period $T$ is determined by the mass conservation, i.e., difference between the ingoing $j_{\text {in }} T$ and outgoing fluxes of particles $j_{\text {out }} T$, see Figs. 3 and 4 . While $j_{\text {in }}$ is a control parameter $j_{\text {in }}=\lambda(1-\lambda), j_{\text {out }}$ is not and it is measured as the time-averaged particle flux through the boundary. $j_{\text {out }}$ depends on the frequency of traffic light $\nu$ and it is associated with the effective right boundary density $\rho_{R}^{e f f}(\nu)$ through the TASEP current-density relation $j_{\text {out }}=\rho_{R}^{\text {eff }}(\nu)\left[1-\rho_{R}^{\text {eff }}(\nu)\right]$ as discussed below. If ingoing and outgoing fluxes are equal,

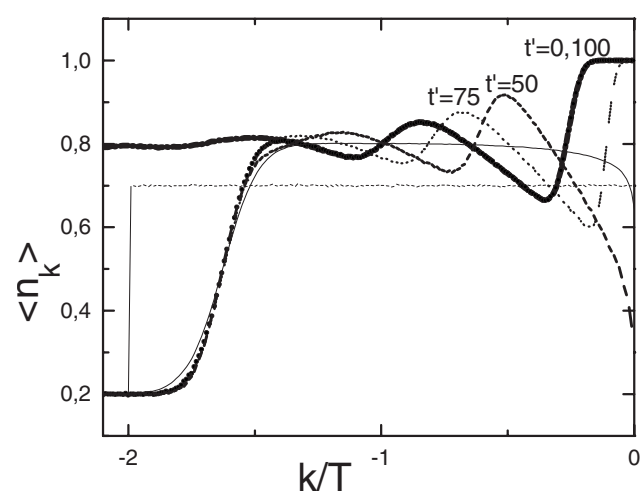

FIG. 2. Snapshots of density profiles at times $t^{\prime}=t-t_{0}$ $=0,50,75,100$ at the coexistence line between LD and HD states $j_{\text {in }}=j_{\text {out }}$. Parameters: $T=100$. The initial configuration of the system is a domain wall $0.2,0.7$ positioned at $k / T=-2$ (dotted piecewise straight line). The system was equilibrated for $t_{0}=5 T=500$ before the measurements, and the averaging over $10^{5}$ histories was done. The thick line shows the density profile after $t=800$ in the system prepared initially in HD state (taken from Fig. 3). The thin line shows the density profile averaged over several periods and many histories after the initial transient period of $5 T$ and it looks (apart from the boundary layer) like the unbiased domain wall in TASEP with constant boundary rates.

the shock only "breathes" around its initial position, see Fig. 2. The density profile behind the shock front develops approximately equidistant sawteethlike structures with the decaying amplitudes, see Fig. 3. The sawteeth profile is changing with time, but it regains its shape after each complete period, so that the shape depends only on the phase

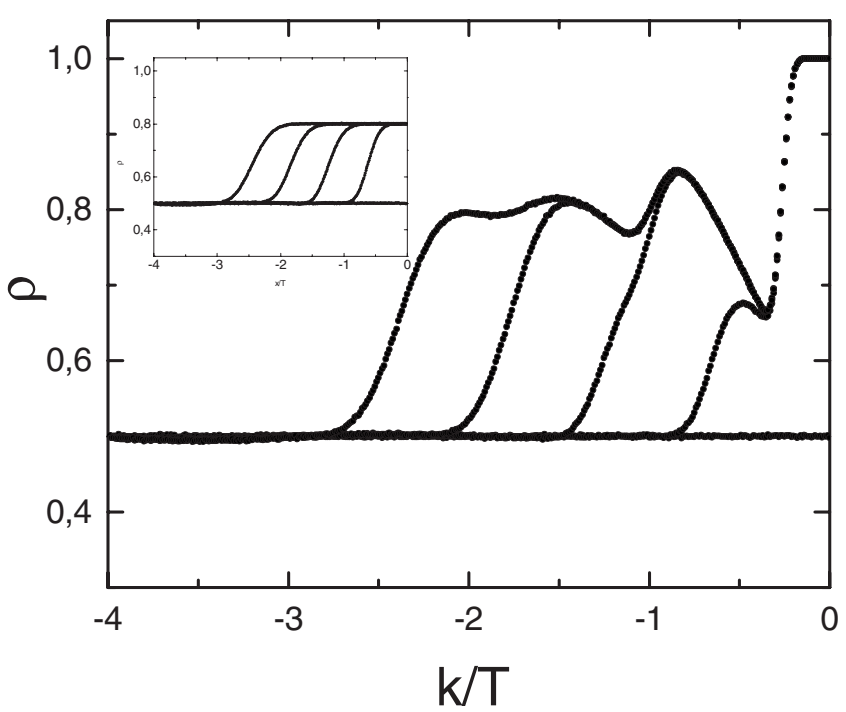

FIG. 3. Snapshots of averaged density profiles $\rho(x, t)$ at $t=0$ [homogeneous state with the density $\left.\left\langle n_{k}\right\rangle=0.5\right]$ and then at equal intervals of time (equal to two periods $T$ ) $t_{i}=200,400,600,800$. Parameters: $\nu=T^{-1}=0.01$, system size 400 , left boundary density 0.5 , averaging is done over $3 \times 10^{5}$ histories. Inset shows the snapshots of density profiles $\left\langle n_{k}\right\rangle$ at times $t=t_{i}$ in the case when instead of traffic light conditions, time-independent boundary conditions are applied with the same effective right boundary density $\rho_{R}$ $=\rho_{R}^{e f f}(\nu) \approx 0.8$. 


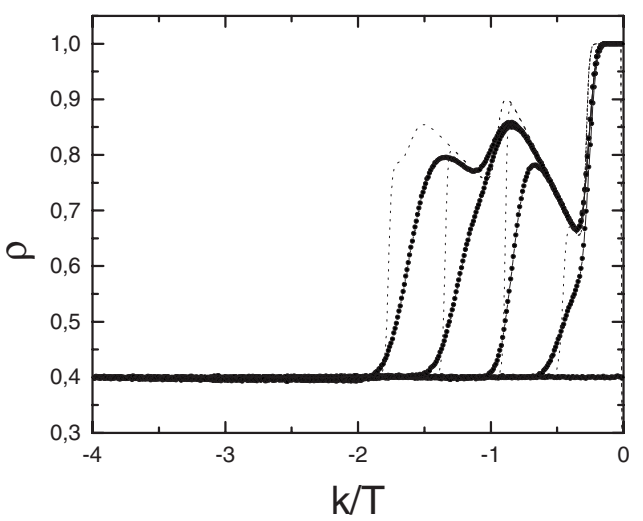

FIG. 4. Same as in Fig. 3 but for an initial homogeneous state with density $\rho(x, 0)=0.4$. Dotted lines show the result on numerical integration of mean-field equations (6) with $p=1$.

$\gamma \subset[0, T]$. Apart from this phase dependence, illustrated, e.g., on Fig. 2, the sawteeth structure behind the shock front depends on frequency $\nu$ (the rescaled sawteeth become sharper with decreasing $\nu$ ) but not on $\lambda$, see Figs. 2-4. The particle density at some distance from the boundary approaches a constant value, $\rho_{R}^{e f f}(\nu)$. This value can be identified with an effective right boundary density in the following sense: the particle system (ASEP) behaves as if it was joined at the origin with the reservoir of particles with the density $\rho_{R}=\rho_{R}^{e f f}(\nu)$. As a function of frequency the averaged stationary flux through the boundary changes monotonically, which implies a monotonic change of $\rho_{R}^{\text {eff }}(\nu)$. In Fig. 5 we report the numerical results for $\rho_{R}^{\text {eff }}(\nu)$ from the Monte Carlo simulations.

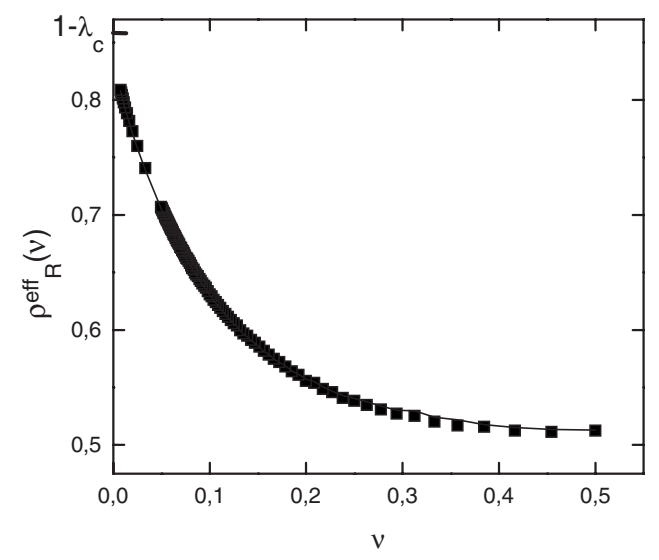

FIG. 5. The averaged stationary density (for parameters chosen equal to effective right boundary density $\rho_{R}^{\text {eff }}$ ) in the TASEP vs the frequency of "traffic light" switch at the right boundary, $\nu=T^{-1}$, from Monte Carlo simulations. The parameters are system size 400, the left boundary density is higher than $1 / 2$, and the averaging is done over 30 histories and over $2 \times 10^{5}$ Monte Carlo steps, after the equilibration. Points show the results of density averages, and the broken line reports estimates from stationary flux measurements.

\section{HYDRODYNAMIC THEORY AND SAWTOOTH STRUCTURE}

\section{A. Limiting cases}

In order to understand the origin of these observations we first argue that if $\lambda \geq 1 / 2$ then the right boundary reservoir density $\rho_{R}^{e f f}(\nu)$ must indeed be equal or larger than $1 / 2$ for any value of $\nu$, as observed: During the "green light" periods $\beta=1$ at most one particle per time unit can exit, while during the "red light" periods $\beta=0$ no particles can exit. For frequencies large compared to the mean attempt rate for particle jumps (which is 1), a particle at the boundary site "sees" the reservoir with equal probability empty or fully occupied, $i r$ respectively of how long it has already stayed at the boundary site. Hence the system behaves like a time-homogeneous system where at each time a particle can exit with the effective rate $\beta^{e f f}=1 / 2$, which corresponds to $\rho_{R}^{\text {eff }}=1-\beta^{\text {eff }}=1 / 2$ in TASEP. So we have $\lim _{\nu \rightarrow \infty} \rho_{R}^{\text {eff }}(\nu)=1 / 2$ which we expect to be a good approximation for all frequencies $\nu \gg 1$ much larger than the jump attempt rate.

On the other hand, in the static case of zero frequency the system relaxes into the high density phase by a back-moving shock if the traffic light cycle starts with $\beta=0$. This leaves the system with a bulk density $\rho=1$. If started with a green traffic light, $\beta=1$, the system reaches the maximal-current phase with bulk density $\rho=1 / 2$ [21]. Continuity in frequency then gives $\rho_{R}^{e f f}(\nu) \geq 1 / 2$ for all frequencies $\nu$ provided that $\lambda \geq 1 / 2$ [27]. If the system is initially in the low density phase, then in the high frequency limit one has again an effective right boundary density of $1 / 2$, leaving the system in the low density phase with a bulk density $\lambda$. In the zerofrequency case the limiting behavior depends again on how the period starts. If $\beta=0$ (red traffic light), the system fills up as described above and $\rho=1$. On the other hand, for $\beta=1$, the system remains in the low density phase with a bulk density $\lambda$.

Notice that neither of these zero-frequency behaviors represents the zero-frequency limit $\nu \rightarrow 0$ shown in Figs. 7 and 8. For this limit we demonstrate in Sec. IV that

$$
\lim _{\nu \rightarrow 0} j\left[\rho_{R}^{e f f}(\nu)\right]=\frac{j_{\max }}{2} \quad \text { and } \rho_{R}^{e f f}(\nu)>\rho^{*} .
$$

This result is based on hydrodynamic limit arguments which are the subject of the following sections.

\section{B. Hydrodynamic limit and mean-field description}

The most basic question to be asked about the dynamics of an interacting particle system is its large-scale behavior, i.e., how macroscopic equations of motion arise from its microscopic dynamics. By suitable coarse-graining of space and time the law of large numbers usually guarantees that stochastic variables, in the present case the particle number in some interval (which under scaling becomes infinite on the microscopic scale, but still infinitesimal on the macroscopic scale), turn into mean values whose temporal evolution satisfy some deterministic evolution equation in rescaled macroscopic time. Moreover, on macroscopic time scales the system is locally stationary, i.e., all fast variables not cap- 
tured in the evolution equation are locally stationary. This fact determines the precise form of the macroscopic equation, provided the stationary distributions are known.

In the case of a conserved quantity the evolution equation is a conservation law of the form

$$
\frac{\partial \rho}{\partial \tau}+\frac{\partial[j(\rho)]}{\partial x}=0
$$

where $x$ is the rescaled space variable and $\tau$ is rescaled macroscopic time $[16,22]$. The quantity $j(\rho)$ is the particle current which on the macroscopic time scales for which Eq. (3) is valid takes its locally stationary value at density $\rho(x, \tau)$. In the case of the ASEP one has $j(\rho)=(p-q) \rho(1-\rho)$. Generally, for driven particle systems with a finite macroscopic current $j(\rho)$ the appropriate hydrodynamic scale is the Euler scale $\tau=t a, x=k a$, where $a \rightarrow 0$ is the lattice constant. For the ASEP investigated here we get the inviscid Burgers equation

$$
\frac{\partial \rho}{\partial \tau}+(p-q) \frac{\partial[\rho(1-\rho)]}{\partial x}=0
$$

on the semiline $x \leq 0$ with traffic light boundary conditions on the right boundary $x=0$. For the TASEP we set $p=1$ and $q=0$.

Notice that in the present case of periodic boundary driving also the period has to be rescaled. The boundary conditions become $\rho(0, \tau)=\frac{1}{2}\{1+\operatorname{sgn}[\sin (2 \pi \Omega t)]\}$ where $\Omega=\nu / a$ is the rescaled frequency. That is, the boundary stays open $\left[\rho\left(0, \tau_{\text {green }}\right)=0\right]$ during green light half-periods $\tau_{\text {green }} \subset\left[0, \frac{T}{2}\right],\left[T, \frac{3 T}{2}\right], \ldots$ and closed $\rho\left(0, \tau_{\text {red }}\right)=1$ during the "red light" half-periods $\tau_{\text {red }} \subset\left[\frac{T}{2}, T\right],\left[\frac{3 T}{2}, 2 T\right], \ldots$. Here we denote by $T$ the complete period $T=1 / \Omega$.

The inviscid Burgers equation is the zero-viscosity limit of the viscous Burgers equation

$$
\frac{\partial \rho}{\partial \tau}+\frac{\partial[\rho(1-\rho)]}{\partial x}=D \frac{\partial^{2} \rho}{\partial x^{2}},
$$

which can be solved in explicit form by a Hopf-Cole transformation for fairly general boundary conditions [23]. For traffic light boundary conditions, however, such a solution is difficult to obtain and we solve the coarse-grained time evolution of the ASEP by numerical integration. To this end we note that the exact microscopic operator equations of motion for the expected particle number $n_{k}$ on site $k$ read

$$
\begin{aligned}
\frac{\partial}{\partial t}\left\langle n_{k}\right\rangle= & p\left\langle n_{k-1}\left(1-n_{k}\right)\right\rangle-q\left\langle n_{k}\left(1-n_{k-1}\right)\right\rangle-p\left\langle n_{k}\left(1-n_{k+1}\right)\right\rangle \\
& +q\left\langle n_{k+1}\left(1-n_{k}\right)\right\rangle .
\end{aligned}
$$

In this equation a one-point function (the expected density $\left.\left\langle n_{k}\right\rangle\right)$ is coupled to a two-point functions (on the right-hand side of the equation), i.e., the equation is not closed. Writing down an exact equation for the two-point functions introduces three-point functions and so on. This infinite hierarchy of equations is not directly tractable and some closure scheme must be employed for further analysis.

In the mean-field approximation for the ASEP, we neglect the correlations and approximate $\left\langle n_{k} n_{k+1}\right\rangle=\left\langle n_{k}\right\rangle\left\langle n_{k+1}\right\rangle$ $=\rho_{k} \rho_{k+1}$, etc. where $\rho_{k}=\left\langle n_{k}\right\rangle$ is an average particle density at

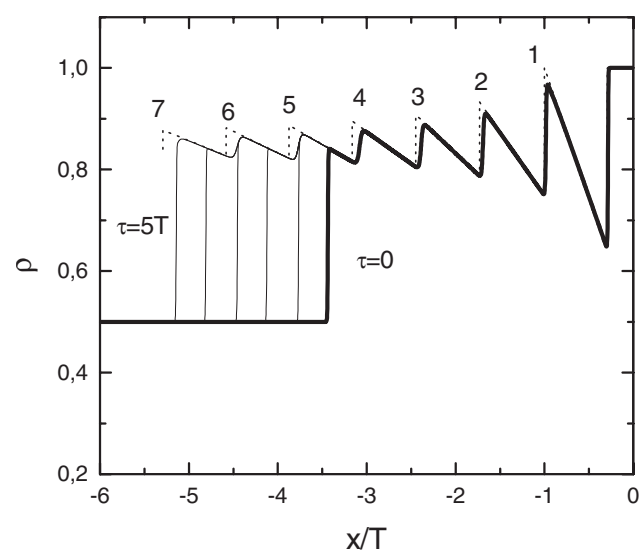

FIG. 6. Results of numerical integration of mean-field equations (6) for system size $N=3000, \nu=T^{-1}=0.002$, with initial condition $\rho(x)=0.5$ after ten full periods (thick curve). Thin curves show the subsequent density profiles $\rho(x, \tau)$ after $1-5$ full periods. The broken line shows the curve $\Gamma$, Eqs. (9)-(12), exact in the limit $\nu \rightarrow 0$.

site $k$. Using this approach for the exact microscopic evolution equation we obtain after some algebra

$$
\begin{aligned}
\frac{\partial}{\partial t} \rho_{k}= & -(p-q)\left[\left(1-2 \rho_{k}\right) \frac{\rho_{k+1}-\rho_{k-1}}{2}\right]+(p+q) \\
& \times\left[\frac{\rho_{k-1}+\rho_{k+1}-2 \rho_{k}}{2}\right] .
\end{aligned}
$$

These equations are complemented with the traffic-light boundary conditions $\rho_{0}(t)=\rho_{R}(t)$ in Eq. (1).

Some comments are in order. First, in the continuum limit one substitutes $k a=x$, with $a \ll 1$ being the lattice constant, e.g., $\rho_{k+1}(t) \rightarrow \rho(x+a, t)$. Taylor-expanding Eq. (6), and using $(p+q=1)$ we obtain, after rescaling time as $\tau=t a$, the Burgers equation

$$
\frac{\partial \rho}{\partial \tau}+(p-q) \frac{\partial[\rho(1-\rho)]}{\partial x}=\frac{a}{2} \frac{\partial^{2} \rho}{\partial x^{2}} .
$$

Therefore the mean-field equation (6) can be viewed as a discretization of the viscous Burgers equation (5), with a constant discretization step $a$, and $D=a^{2} / 2$. In fact, for numerical integration of the Burgers equation (5) with the diffusion coefficient $D$, we shall be using the discretization scheme (6) with $p=1, q=0$, discretization step $a=1$, and the coefficient $2 D$, instead of $(p+q)$ in front of the discrete second derivative.

Second, for the case of weak hopping asymmetry $\lim _{a \rightarrow 0} a(p-q)=1$ one can obtain Eq. (5) from Eq. (6) by diffusive rescaling $\tau=t a^{2} / D, x^{\prime}=x a^{2} / D$ in the hydrodynamic limit $a \rightarrow 0$. In this case, the density does not evolve into shocks. A stationary traveling wave solution is a hyperbolic tangent with a step width proportional to the square root of the viscosity. Such smoothening of a shock may also be expected from the integration of the discrete mean-field equation (6). In Fig. 6 we show the results obtained from numerical integration of the mean-field equations (6) when a traffic light boundary is present at the origin. We see that the density profiles $\rho(x, \tau)$ display a sawtooth structure which re- 


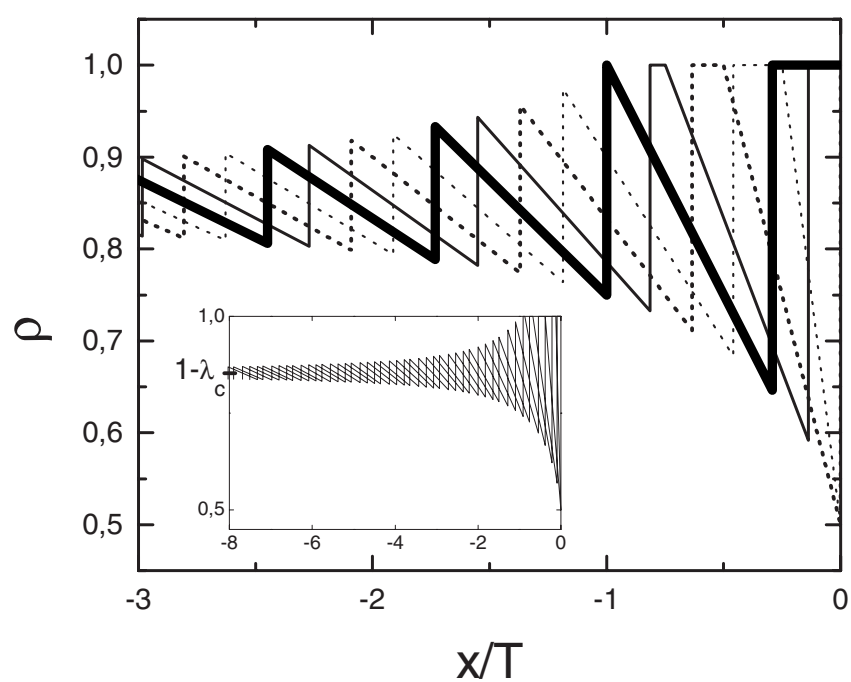

FIG. 7. The universal curve $\Gamma$ (solid curve) at the beginning of the green light period $\tau=0, T, 2 T, \ldots$ (thick curve) and for intermediate $\tau$ values $\tau / T=1 / 4,1 / 2,3 / 4$ (broken, thick broken, thin curves, respectively). Inset shows $\Gamma$ snapshots over a larger scale. Note that microscopically the snapshots of average density profiles during the green light period $\rho_{R}=0$ do not have discontinuity at the first site, but a boundary layer involving many sites, interpolating between $\rho=0.5$ and $\rho_{R}=0$. However, the $\Gamma$ snapshots show the limiting density profiles, rescaled by the period $T$, in the limit when $T \rightarrow \infty(\nu=+0)$. The boundary layer vanishes in this limit leading to a discontinuity at $x=0$.

sembles the one observed for ASEP (see Fig. 3).

As a warning to readers not familiar with hydrodynamic scaling, we remark that the agreement between the meanfield equation for the ASEP in the continuum limit and the rigorously derived Burgers equation is purely coincidental. It arises from the fact that for the ASEP the stationary distribution has no correlations and hence the mean-field equations become exact. In general lattice gases, e.g., in the KatzLebowitz-Spohn model [5], one has correlations and a simple-minded mean-field approach neglecting all correlations would produce a macroscopic equation that is in general not even qualitatively correct.

\section{Stationary sawtooth structure}

The time-periodic sawtooth solution shown in Fig. 6 is a shock analog for the case of periodically changing boundary conditions which we now describe in detail. To this regard, we take the analytically tractable Burgers case (4) as a concrete example for explicit computation. The results for stationary (periodically repeating) solutions obtained in this case are expected to be valid also for a generic conservation law equation with convex $j(\rho)$.

Looking at snapshots of density profiles $\rho(x, t+m T)$ at times differing by multiples $m T$ of a period, one notes that they fill some universal curve $\Gamma$, which has a characteristic sawtooth shape, see Fig. 6 . The curve $\Gamma_{\gamma}(x, \tau)=\Gamma_{\gamma}(x, \tau+T)$ "breathes" and returns to its original form after a full time period $T$, see Fig. 7. The index $\gamma$ denotes a phase $0 \leq \gamma<T$ at which the snapshot of $\Gamma$ is taken, with respect to the begin-

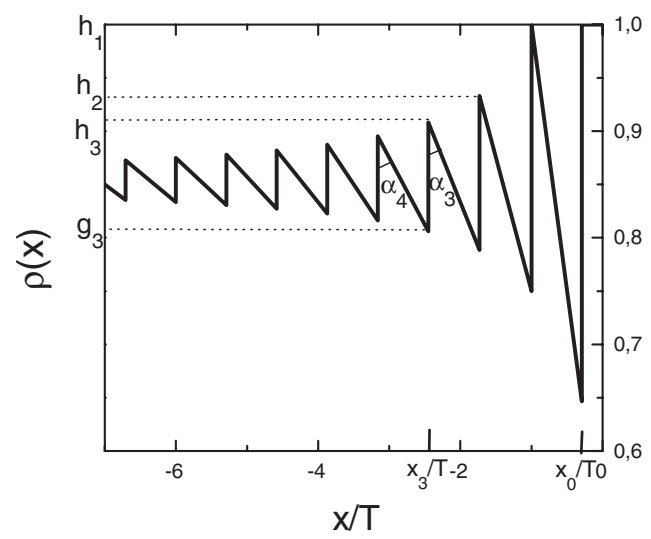

FIG. 8. The universal curve $\Gamma$ (solid curve) at the beginning of the green light period and variables $g_{k}, h_{k}, \alpha_{k}$ used in the analysis, Eqs. (9)-(12).

ning of a green light interval. In the following we shall set $\gamma=0$ and omit $\gamma$ for brevity of notation.

First, we describe the curve $\Gamma$ and then prove its periodicity in time. The curve consists of an infinite number of sawteeth with heights decreasing away from the boundary. We denote the height, the base, and the coordinate of the $k$ th sawtooth at the beginning of green light periods by $h_{k}^{0}, g_{k}^{0}$, and $x_{k}^{0}$, respectively, and the sawtooth angle by $\alpha_{k}^{0}$ as shown in Fig. 8. Each sawtooth is bounded by a shock discontinuity on the left and by a rarefaction wave on the right except the sawtooth $k=0$ bounded on the right by a jam (caused by a just finished red light period). Shock discontinuities move with the velocities given by the Rankine-Hugoniot condition

$$
v_{\text {shock }}(k)=\frac{j\left(h_{k}\right)-j\left(g_{k}\right)}{h_{k}-g_{k}},
$$

where $j(\rho)$ is a flux function from Eq. (3). In the framework of stochastic driven systems $j(\rho)$ is called the current-density relation or fundamental diagram. Shapes of rarefaction waves are also determined by $j(\rho)$. In the following we shall consider a specific example (4) However, we expect that our main results (30)-(32) are applicable for arbitrary convex function $j(\rho)$.

For the specific case of the inviscid Burgers equation, $h_{k}^{0}$, $g_{k}^{0}, x_{k}^{0}$, and $\alpha_{k}^{0}$ for $k>0$ are given by

$$
\begin{gathered}
h_{k}^{0}=\frac{1}{2}\left(1+\left(1-2 \lambda_{c}\right) \sqrt{1+\frac{1}{k}}\right), \\
g_{k}^{0}=\frac{1}{2}\left(1+\left(1-2 \lambda_{c}\right) \sqrt{1-\frac{1}{k+1}}\right), \\
-x_{k}^{0}=k T\left(1-2 \lambda_{c}\right) \sqrt{1+\frac{1}{k}}, \\
\tan \left(\alpha_{k}^{0}\right)=2 k T,
\end{gathered}
$$

and for $k=0$

$$
-x_{0}^{0}=2 \lambda_{c} T,
$$




$$
g_{0}^{0}=\frac{1}{2}+\lambda_{c},
$$

where $\lambda_{c}=(2-\sqrt{2}) / 4 \approx 0.146$ satisfies $\lambda_{c}\left(1-\lambda_{c}\right)=1 / 8$. For $k$ $\gg 1$, both $h_{k}^{0} \approx 1-\lambda_{c}+\frac{1-2 \lambda_{c}}{4 k}$ and $g_{k}^{0} \approx 1-\lambda_{c}-\frac{1-2 \lambda_{c}}{4 k}$ approach the limiting value $1-\lambda_{c}$ which is therefore the limit of $\rho(x, \tau)$ as $x \rightarrow-\infty$. This value may be identified with the effective time-independent boundary density $\rho_{R}^{\text {eff }}$. For rigorous definition of the boundary density see [19]. Here we only stress that the underlying particle system (ASEP) behaves as if it was joined at the origin with the reservoir of particles with the density $\rho_{R}^{e f f}$. Correspondingly, the average flux through the boundary is

$$
\left\langle j_{\text {out }}\right\rangle=j\left(\rho_{R}\right)=j\left(1-\lambda_{c}\right)=\frac{1}{8}=\frac{j_{\max }}{2} .
$$

In the following we prove that $\Gamma$ is a periodic function of time $\Gamma(\tau)=\Gamma(T+\tau)$. In this respect it is sufficient to show that the heights and positions of all sawteeth will be the same after time $T$. However, since all sawteeth shocks discontinuities move to the left $\partial x_{n}(\tau) / \partial \tau<0$ [this follows from $h_{n}(\tau)$ $>g_{n}(\tau)>1 / 2$ and Eq. (8)], sawteeth cannot return to their original places. Instead, after time $T$ a sawtooth $k$ will take the place of a former sawtooth $k+1$, i.e., $h_{k}(T)=h_{k+1}^{0}, g_{k}(T)$ $=g_{k+1}^{0}$, and $x_{k}(T)=x_{k+1}^{0}$, for all $k$, see Fig. 7. At the boundary, the structure with a jam at position $x_{0}^{0}$ must be regenerated after a complete green light $\tau \subset[0, T / 2]$ and red right $\tau \subset[T / 2, T]$ period. To proceed, note that the inviscid Burgers equation (4) has two basic solution types: (a) between two consecutive homogeneous states $\rho_{-}<\rho_{+}$a shock discontinuity is formed moving with velocity $v_{\text {shock }}=1-\rho_{-}-\rho_{+}$and (b) between two consecutive homogeneous states $\rho_{-}>\rho_{+}$a rarefaction wave $\rho(x, \tau)=\rho(x / \tau)=1 / 2-x /(2 \tau)$ is formed. This information is enough to predict the evolution of a sawteeth structure in Fig. 8, consisting of shocks and rarefaction waves. In particular, the shock velocity of a $k$ th sawtooth

$$
\frac{\partial x_{k}}{\partial \tau}=1-h_{k}(\tau)-g_{k}(\tau)
$$

and

$$
\frac{\partial}{\partial \tau} \tan \left[\alpha_{k}(\tau)\right]=2
$$

In the following we shall explicitly indicate time-dependent quantities, e.g., $h_{k}(\tau), \alpha_{k}(\tau)$ while $h_{k}^{0}, \alpha_{k}^{0}$ will denote their initial values $h_{k}^{0}=h_{k}(0)$, etc., given by Eqs. (10)-(12). The time interval $0 \leq \tau \leq T$ will be considered. For $k>0$ Eqs. (16) and (12) are trivially solved: $\tan \alpha_{k}(\tau)=2(\tau+k T)$. For $h_{k}(\tau), g_{k}(\tau)$ we can write the equations

$$
\begin{gathered}
\frac{-x_{k}(\tau)}{h_{k}(\tau)-\frac{1}{2}}=\tan \alpha_{k}(\tau)=2(\tau+k T), \\
\frac{-x_{k}(\tau)}{g_{k}(\tau)-\frac{1}{2}}=\tan \alpha_{k+1}(\tau)=2[\tau+(k+1) T] .
\end{gathered}
$$

Multiplying Eq. (17) by $h_{k}(\tau)-\frac{1}{2}$, differentiating with respect to $\tau$, and using Eq. (15) we obtain

$$
2(\tau+k T) \frac{\partial h_{k}(\tau)}{\partial \tau}+h_{k}(\tau)-g_{k}(\tau)=0
$$

Analogously, for $g_{k}(\tau)$ we get an ordinary differential equation

$$
2[\tau+(k+1) T] \frac{\partial g_{k}(\tau)}{\partial \tau}+g_{k}(\tau)-h_{k}(\tau)=0
$$

with the initial conditions $g_{k}(0)=g_{k}^{0}, h_{k}(0)=h_{k}^{0}$. These equations can be integrated to give

$$
\begin{gathered}
h_{k}(\tau)=h_{k}^{0}+\left(h_{k}^{0}-g_{k}^{0}\right)(k+1)\left\{\sqrt{\frac{k T}{k T+\tau} \frac{(k+1) T+\tau}{(k+1) T}}-1\right\}, \\
g_{k}(\tau)=g_{k}^{0}+\left(h_{k}^{0}-g_{k}^{0}\right) k\left\{\sqrt{\frac{k T+\tau}{k T}} \sqrt{\frac{(k+1) T}{(k+1) T+\tau}}-1\right\} .
\end{gathered}
$$

In particular, at time $\tau=T$

$$
\begin{aligned}
& h_{k}(T)=h_{k}^{0}+\left(h_{k}^{0}-g_{k}^{0}\right)\{\sqrt{k(k+2)}-k-1\}, \\
& g_{k}(T)=g_{k}^{0}+\left(h_{k}^{0}-g_{k}^{0}\right) k\left\{\frac{k+1}{\sqrt{k(k+2)}}-1\right\} .
\end{aligned}
$$

It can be verified that $\alpha_{k}(T)=\alpha_{k+1}^{0}, h_{k}(T)=h_{k+1}^{0}$, and $g_{k}(T)$ $=g_{k+1}^{0}$, thus ensuring the regeneration of the curve $\Gamma$ after a period $T$. Special attention should be given to the boundary region $k=0$. The equation for $g_{0}(\tau)$ reads

$$
\frac{-x_{0}(\tau)}{g_{0}(\tau)-\frac{1}{2}}=2(T+\tau) \text {. }
$$

Multiplying by the denominator and differentiating with respect to $\tau$ we obtain $2(\tau+T) \frac{\partial g_{0}(\tau)}{\partial \tau}+2\left[g_{0}(\tau)-\frac{1}{2}\right]=-\frac{\partial x_{0}(\tau)}{\partial \tau}$. At point $x_{0}(\tau)$ there is a jump, $\rho\left[x_{0}(\tau)-0, \tau\right]=g_{0}(\tau)$ and $\rho\left[x_{0}(\tau)+0, \tau\right]=1$ at the right, consequently

$$
\frac{\partial x_{0}}{\partial \tau}=\frac{-g_{0}(\tau)\left[1-g_{0}(\tau)\right]}{1-g_{0}(\tau)}=-g_{0}(\tau) .
$$

Substituting, we obtain

$$
2(\tau+T) \frac{\partial g_{0}(\tau)}{\partial \tau}+g_{0}(\tau)-1=0 .
$$

Solving the latter with the initial condition $g_{0}(0)=g_{0}^{0}$, we get

$$
g_{0}(\tau)=1+\left(g_{0}^{0}-1\right) \sqrt{\frac{T}{T+\tau}},
$$

describing the shock propagation during $0 \leq \tau \leq T$. Alongside, the jam joining the boundary will start to dissolve by rarefaction wave $\rho(x, \tau)=\frac{1}{2}-\frac{x}{2 \tau}$ during the green light period $0 \leq \tau \leq \frac{T}{2}$. During the red light period $\frac{T}{2} \leq \tau \leq T$ a new jam appears at the boundary $x=0$ and propagates inside. We shall denote its coordinate by $x_{G}(\tau), x_{G}\left(\frac{T}{2}\right)=0$. The base of the new jam, denoted by $G(\tau), G\left(\frac{T}{2}\right)=\frac{1}{2}$ will obey 


$$
\frac{-x_{G}(\tau)}{G(\tau)-\frac{1}{2}}=2 \tau
$$

Using $\partial x_{G}(\tau) / \partial \tau=-G(\tau)$, we obtain an equation $2 \tau \frac{\partial G(\tau)}{\partial \tau}$ $+G(\tau)-1=0$, solved by $G(\tau)=1-\frac{1}{2} \sqrt{\frac{T / 2}{\tau}}$ for $\frac{T}{2} \leq \tau \leq T$. At time $\tau=T$ one has $G(T)=1-\frac{1}{2 \sqrt{2}}=\frac{1}{2}+\lambda_{c}=g_{0}^{0}$, restoring the initial shape at $\tau=0$, see Eq. (13). $g_{1}^{0}$ is determined from the solution (21), $g_{0}(T)=g_{1}^{0}$. At time $\tau=T$ the jam, which was initially at position $x_{0}^{0}$, reaches point $x_{1}^{0}$, consequently $h_{1}^{0}=1$. The remaining relations (9)-(11) are obtained recurrently from Eqs. (19) and (20). Thus the proof of periodicity of the sawtooth structure is completed.

\section{Steady-state selection}

In the previous section we have proved stationarity of the sawtooth state under periodic driving, but we did not address the question whether this stationary state is actually reached for any initial state characterized by the initial density $\lambda$. In order to investigate this problem of steady-state selection we consider an initial state consisting of a homogeneous state on the left $\rho_{-}(x)=\lambda$ and a sawtooth structure on the right, joined by a shock, and demonstrate that it is analogous to a shock between the two homogeneous states $\rho_{-}(x)=\lambda$ and $\rho_{+}(x)=1$ $-\lambda_{c}$.

The latter shock moves with the velocity $v=[j(\lambda)-j(1$ $\left.\left.-\lambda_{c}\right)\right] /\left(1-\lambda-\lambda_{c}\right)=\lambda_{c}-\lambda$, and will travel a distance $\left(\lambda_{c}\right.$ $-\lambda) T$ after time $T$. In particular, the shock will be stationary for $\lambda=\lambda_{c}$. Let us prove this feature for the shock between the homogeneous state $\rho_{-}(x)=\lambda_{c}$, for $x<x_{n}$ and the sawtooth structure with $n$ complete sawteeth $\rho_{+}(x)=\Gamma$, for $x_{n}<x<0$. Heights of all sawteeth $h_{k}, k \leq n$ will satisfy Eq. (17), but the velocity for the $n$th sawtooth satisfies

$$
\frac{\partial x_{k}}{\partial \tau}=\frac{h_{k}(\tau)\left[1-h_{k}(\tau)\right]-\lambda_{c}\left(1-\lambda_{c}\right)}{h_{k}(\tau)-\lambda_{c}}=1-h_{k}(\tau)-\lambda_{c} .
$$

Multiplying Eq. (17) for $k=n$ by $h_{n}(\tau)-\frac{1}{2}$, differentiating with respect to $\tau$, and using Eq. (22) we obtain $2(\tau$ $+n T) \frac{\partial h_{n}(\tau)}{\partial \tau}+h_{n}(\tau)-\lambda=0$, whose solution is $h_{n}(\tau)=\lambda_{c}+\left(h_{n}^{0}\right.$ $\left.-\lambda_{c}\right) \sqrt{\frac{n T}{n T+\tau}}$. We need to prove that the shock will return to the original position one after time $T$, i.e., $\int_{0}^{T} \frac{\partial x_{n}}{\partial \tau} d \tau=0$. Substituting the solution for $h_{n}(\tau)$ into Eq. (22), and integrating over the period, we have

$$
\int_{0}^{T} \frac{\partial x_{n}}{\partial \tau} d \tau=\left(2 \lambda_{c}-1\right) T+2 T\left(h_{n}^{0}-\lambda_{c}\right)[\sqrt{n(n+1)}-n]=0 .
$$

Substitution (9) satisfies the above equation.

Analogously, one proves that the shock between $\lambda \neq \lambda_{c}$ and the sawtooth structure, after period $T$, will advance if $\lambda>\lambda_{c}$ or retreat toward the boundary if $\lambda<\lambda_{c}$. The shock position after time $T$ is determined by a balance equation. Namely, the extra mass gained by the shock, $\Delta M$ $=\int_{-\infty}^{0}[\rho(x, T)-\rho(x, 0)] d x$, is equal to the difference between the ingoing and outgoing currents $\left(j_{\text {in }}-j_{\text {out }}\right) T=[j(\lambda)-j(1$ $\left.\left.-\lambda_{c}\right)\right] T$,

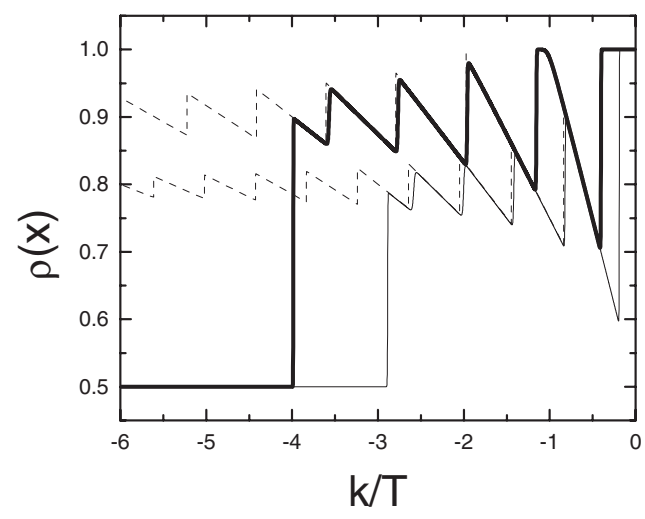

FIG. 9. Sawteeth density profiles for different fractions of the green signal $f=\tau_{\text {green }} / T=0.35,0.65$. Thick $(f=0.35)$ and thin $(f$ $=0.65)$ curves show respective density profiles $\rho(x, \tau)$ from meanfield equations (6) evolving from the initial condition $\rho(x, 0)=0.5$ after ten full periods. In MF calculations, $\nu=T^{-1}=0.001$. Broken lines show the limiting curves $\Gamma(f)$, exact in the limit $\nu \rightarrow 0$. The sawteeth heights are given by Eq. (24). All the curves are shown at the moment of time when the red light turns off.

$$
\Delta M=\left(j_{\text {in }}-j_{\text {out }}\right) T=\left[j(\lambda)-j\left(1-\lambda_{c}\right)\right] T .
$$

The explicit calculations are not illuminating and are omitted for brevity. As a guide to the eye, see Fig. 6. The most rapid progression of the sawtooth structure is achieved for $j_{\text {in }}$ $=j_{\max }=1 / 4$ for which the number of sawteeth $n$ increases roughly by two each five cycles, see Fig. 6.

The variations of the boundary density considered in this paper so far [Eq. (1)] followed a square wave in which the signal is green for a fraction $f=\tau_{\text {green }} / T=\frac{1}{2}$ of the period. What happens if we vary the fraction $f$ between 0 and 1 ? While the cases $f=0$ and 1 are obvious and were discussed before Eq. (2), the general case $0<f<1$ produces a sawteeth structure $\Gamma(f)$, qualitatively similar to the one for $f=1 / 2$, compare Figs. 8, 6, and 9. The limiting curve $\Gamma(f)$ converges in the bulk to the value $1-\lambda(f)=(1+\sqrt{1-f}) / 2$, determined by the outgoing flux in the vanishing frequency limit $\left\langle j_{\text {out }}(f)\right\rangle=f j_{\max }$, see the discussion after Eq. (30). Note that $\lambda(f)$ satisfies $\lambda(f)[1-\lambda(f)]=\left\langle j_{\text {out }}(f)\right\rangle=f / 4$. Analytic analysis for arbitrary $f$ can be carried out analogously to the $f=1 / 2$ case. In particular, the heights of the sawteeth $h_{n}^{0}(f)$ are determined by the solution of Eq. (23) where $\lambda_{c}$ is substituted by $\lambda(f)=(1-\sqrt{1-f}) / 2$,

$$
h_{n}^{0}(f)=\frac{1}{2}+\frac{\sqrt{1-f}}{2} \sqrt{1+\frac{1}{n}},
$$

valid for $n>0$ such that $h_{n}^{0}(f) \leq 1$. This is always the case if $f \geq 1 / 2$ (the duration of the green signal is larger then the duration of the red signal). In the opposite case, $f<1 / 2$, additional plateau appear with the saturated density $\rho=1$ on the curve $\Gamma(f)$, apart from the very first one caused by the red traffic light at the boundary, see upper curve in Fig. 9. A thorough analysis of these more complicated structures is beyond the scope of the present paper. As a guide for an eye, see Fig. 9, where the curves $\Gamma(f)$ for $f=0.35,0.65$ are shown. 
Let us stress once more the universality aspect of the limiting curve $\Gamma$ shown in Figs. 7 and 8. The shape of the curve is independent of $T$ provided that $T$ is sufficiently large, $T^{-1}=\nu \rightarrow 0$. It also does not depend also on ASEP bulk rates $p, q$ provided that $p>q$ (drive toward the right boundary), since $\Gamma$ is described by Eq. (4). Qualitatively, $\Gamma$ does not depend on relative duration of the green light period with respect to the whole period $T$ (equal to $1 / 2$ in the present study), as argued in the previous paragraph. Moreover, for other models with the convex current-density relation $j(\rho)$ and traffic light boundary conditions we expect the existence of a similar curve with sawtooth structure, with modeldependent shape of the sawteeth. The distance between nearest sawteeth is determined by $j(\rho)$, see Eq. (31). The sawteeth curve in the bulk will converge to a value $\rho_{R}^{\text {eff }}(\nu)$, determined by averaged outgoing flux through $\left\langle j_{\text {out }}\right\rangle$ $=j\left[\rho_{R}^{e f f}(\nu)\right]$. The outgoing flux in the limit $\nu \rightarrow 0$ will be given by Eq. (30).

\section{FINITE SIZE ASEP WITH TRAFFIC LIGHT BOUNDARY CONDITIONS AT ONE OR BOTH BOUNDARIES}

Here we discuss the stationary behavior of a sufficiently large but finite system of size $N$ with open boundaries. It is intuitively clear that the periodically changing conditions at a boundary will generate a sawteeth structure with a typical sawtooth size $\Delta(\nu) \approx \frac{1}{\nu}\left(\frac{\partial j}{\partial \rho}\right)_{\rho=\rho_{R}^{e f f}(\nu)}$, see the paragraph preceding Eq. (31). We shall consider the case $N \gg \Delta$. If $N \lesssim \Delta$, then one boundary will influence another one during a periodic cycle.

First, recall the well-known results for the TASEP model with time-independent boundary rates, solved exactly in $[13,14]$. In the TASEP, a particle can be injected at first site $k=1$ from the left boundary reservoir with the rate $\alpha$ and be extracted from the last site $k=N$ with the rate $\beta$. This corresponds to coupling at the left with the reservoir of particles with density $\rho_{L}=\alpha$ and on the right with the reservoir of particles with the density $\rho_{R}=1-\beta$. In the range of parameters $0 \leq \alpha, \beta \leq 1$ the stationary states are characterized by average homogeneous particle distribution in the bulk with density $\rho_{\text {stat }}\left(\rho_{L}, \rho_{R}\right)$. The stationary densities obey an extremal principle for the stationary flux [5],

$$
j_{\text {stat }}=\left\{\begin{array}{ll}
\min _{\left[\rho_{L}, \rho_{R}\right]} j(\rho) & \text { if } \rho_{L}<\rho_{R} \\
\max _{\left[\rho_{L}, \rho_{R}\right]} j(\rho) & \text { if } \rho_{L}>\rho_{R}
\end{array},\right.
$$

which, being applied for the case of ASEP $j(\rho)=\rho(1-\rho)$, yields three different phases,

Low density $(\mathrm{LD}) \rho_{\text {stat }}=\rho_{L}$ for $\rho_{L}=\alpha<1 / 2, \rho_{R}<1-\rho_{L}$,

High density(HD) $\rho_{\text {stat }}=\rho_{R}$ for $\rho_{R}>1 / 2, \rho_{R}>1-\rho_{L}$,

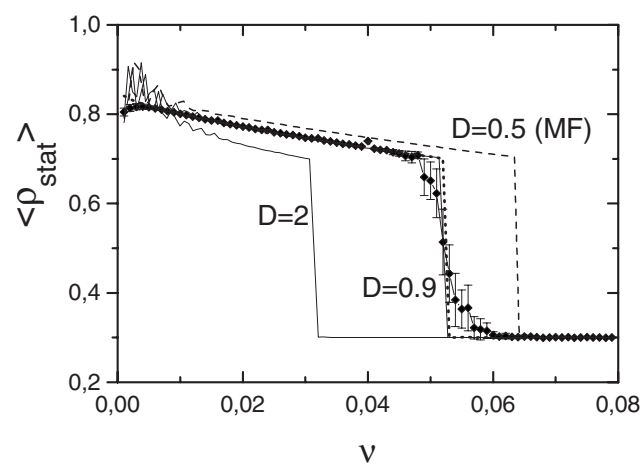

FIG. 10. Stationary density as function of the frequency $\nu$ for fixed left boundary density $\rho_{L}=0.3$, from Monte Carlo simulations (diamonds with error bars), and from integration of Burgers equation (7) with $D=2,0.9$ (solid lines) and $D=0.5$ (broken line). The dotted line shows $\left\langle\rho_{\text {stat }}\right\rangle$ computed from stationary flux measurements. Fluctuations at small frequencies are due to finite size effects.

$$
\text { Max. current }(\mathrm{MC}) \rho_{\text {stat }}=1 / 2 \text { for } \rho_{L}>1 / 2, \rho_{R}<1 / 2 \text {. }
$$

In the case of fixed left boundary conditions $\rho_{L}=$ const and periodically changing right boundary conditions (e.g., traffic light boundary conditions) the $\rho_{R}$ in Eqs. (25)-(28) has to be replaced by the effective boundary density $\rho_{R}^{e f f}(\nu)$ and $j_{\text {stat }}, \rho_{\text {stat }}$ by a time-averaged flux and density in a stationary state $\left\langle j_{\text {stat }}\right\rangle,\left\langle\rho_{\text {stat }}\right\rangle$, respectively. Note that since $\rho_{R}^{\text {eff }}(\nu)$ $\geq 1 / 2$ for any $\nu$, only LD and HD phases can be observed.

In particular, Eqs. (25)-(28) with the latter substitution predicts a discontinuous change of stationary density from $\left\langle\rho_{\text {stat }}\right\rangle=\rho_{L}<1 / 2$ (LD phase) to $\left\langle\rho_{\text {stat }}\right\rangle=\rho_{R}^{e f f}(\nu)$ (HD phase) at a transition point $1-\rho_{L}=\rho_{R}^{e f f}(\nu)$. Indeed, keeping $\rho_{L}$ fixed and changing $\nu$, one observes this phase transition at the predicted point, see Fig. 10.

Analogously, keeping fixed right boundary conditions $\rho_{R}$ $=$ const and applying traffic light boundary conditions at the left boundary, one has to define the effective left boundary density $\rho_{L}^{e f f}(\nu)$. The latter, due to particle-hole symmetry of the TASEP, is given simply by

$$
\rho_{L}^{e f f}(\nu)=1-\rho_{R}^{e f f}(\nu)
$$

Consecutively, the $\rho_{L}^{\text {eff }}(\nu)$ varies between $\rho_{L}^{\text {eff }}(\nu \rightarrow+0)=\lambda_{c}$ to $\rho_{L}^{e f f}(\nu \rightarrow \infty)=1 / 2$.

Finally, applying traffic light boundary conditions at both boundaries with the frequencies $\nu$ and $\nu^{\prime}$ on the left and on the right, respectively, effective boundary reservoirs $\rho_{L}^{\text {eff }}(\nu)$ at the left and $\rho_{R}^{e f f}\left(\nu^{\prime}\right)$ at the right are created. Again, one finds the phase diagram applying the rule (25) with the replacements $\rho_{L} \rightarrow \rho_{L}^{\text {eff }}(\nu), \rho_{R} \rightarrow \rho_{R}^{\text {eff }}\left(\nu^{\prime}\right)$. Taking into account Eq. (29), one predicts the LD [Eq. (26)] phase $\left\langle\rho_{\text {stat }}\right\rangle$ $=\rho_{R}^{\text {eff }}(\nu)$ for $\nu>\nu^{\prime}$ and the HD [Eq. (27)] phase $\left\langle\rho_{\text {stat }}\right\rangle$ $=\rho_{L}^{e f f}(\nu)=1-\rho_{R}^{e f f}(\nu)$ for $\nu<\nu^{\prime}$. Due to the range of variance of the effective boundary densities $\rho_{L}^{\text {eff }}(\nu)<1 / 2,1 / 2$ $<\rho_{R}^{\text {eff }}(\nu)$, the maximal current phase (28) cannot be reached except at one point, see Fig. 11. 


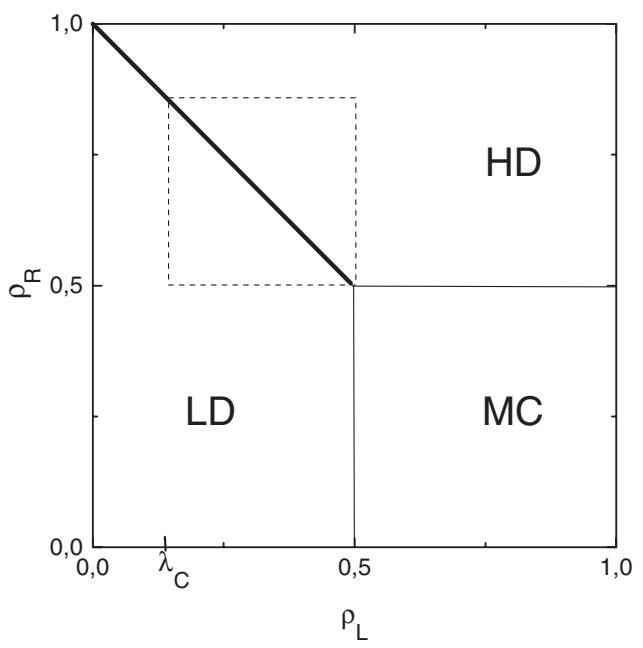

FIG. 11. Phase diagram of ASEP with open boundaries, illustrating Eqs. (26)-(28). Solid line indicates a discontinuous LD/HD transition. Dashed line borders the region reachable by applying the traffic light boundary conditions with arbitrary frequencies at both boundaries.

In addition to Monte Carlo simulations, we integrated numerically mean-field equations (6). In the limit of infinitely small frequencies $\nu \rightarrow 0$ the solution of the mean-field equations converges to the solution of the inviscid Burgers equation, see Figs. 6 and 9. For finite frequencies $\nu>0$, we observe qualitative agreement between mean-field and Monte Carlo density profiles, see Fig. 4. However, the mean-field solution apparently fails to predict the exact location of the phase transition frequency, see the curve with $D=0.5$, marked MF, in Fig. 10. Indeed, as argued after Eq. (7), the mean-field equation can be viewed as a discretization of the viscous Burgers equation with the diffusion coefficient $D$ $=0.5$. The mean-field approach fails quantitatively because it neglects correlations which are present in the sawtooth state. On the other hand, numerical integration of the Burgers equation (5), keeping the diffusion coefficient $D$ a free parameter, shows that the effective boundary density (and consequently the critical frequency) depends on $D$. Manipulating $D$, one can obtain a better agreement with the Monte Carlo simulations, see Fig. 10. It might seem from Fig. 10 that the choice $D=0.9$ fits the Monte Carlo data well. However, the deviation between Monte Carlo data and the Burgers equation with $D=0.9$ for small frequencies $\nu$ is substantial and it can be seen by comparing graphs of type Fig. 10 for different $\rho_{L}$ (not shown).

\section{CONCLUSIONS}

We have provided a hydrodynamic description of the semi-infinite ASEP with traffic light boundary conditions. We find a time-periodic stationary sawtooth solution which is described in detail. We have also addressed the question of steady-state selection, starting from some initial density $\lambda$. The picture that emerges is similar to that of the usual ASEP with constant effective reservoir density that we have determined. Despite the sawtooth structure of the solution, the time averaged density at sufficiently large distance from the boundary is given by $\bar{\rho}(x, t)=\rho^{e f f}$, both in the low and high density regime. Indeed, considering the motion of the shock as an effective single-particle problem in an external potential $[24,25]$ this observation is reminiscent of the motion of a Brownian particle in a periodically driven stochastic system [26]. Our result shows that effective potentials may arise also in interacting many-body systems as a result of periodic driving.

Our derivation is based on the ASEP as a specific example, but remains valid for generic driven diffusive systems with a convex current-density relation. There are several quantitative conclusions that one can draw from the exact hydrodynamic treatment presented above. First, note that the average outgoing flux, see Eq. (14), $\left\langle j_{\text {out }}\right\rangle=1 / 8$ is two times smaller than the maximal flux $\max j(\rho)=j_{\max }=1 / 4$. The relation

$$
\left\langle j_{\text {out }}\right\rangle=\frac{j_{\max }}{2}
$$

is not a casual, but a rather generic one: during the red light periods, $\left\langle j_{\text {out }}\right\rangle_{\text {red }}=0$, and an extended jam at the boundary forms. During the green light period, $\left\langle j_{\text {out }}\right\rangle_{\text {green }}=j_{\max }$ because the outflow from a jam is governed by a maximization principle $\left\langle j_{\text {out }}\right\rangle_{\text {green }}=\max _{\rho \subset[0,1]} j(\rho)=j_{\max }[4]$. Per full period, one obtains Eq. (30).

Second, the distance between neighboring sawteeth rapidly approaches a constant, $\left|x_{k+1}-x_{k}\right| \approx\left(1-2 \lambda_{c}\right) T$ for $k \gg 1$, as follows from Eq. (11). The value of the constant has a simple physical origin: maximum and minimums of sawtooth structure $\Gamma, h_{k}$, and $g_{k}$ approach the effective boundary density value $h_{k}, g_{k} \approx 1-\lambda_{c} \pm O(1 / k)=\rho_{R} \pm O(1 / k)$. Hence the velocities of the discontinuities for large $k$ approach the group velocity $v_{\text {group }}\left(\rho_{R}\right)=\lim _{k \rightarrow \infty} \frac{h_{k}(\tau)\left[1-h_{k}(\tau)-g_{k}(\tau)\left[1-g_{k}(\tau)\right]\right.}{h_{k}(\tau)-g_{k}(\tau)}$ $=(\partial j / \partial \rho)_{\rho=\rho_{R}}$. By the periodicity $\Gamma(0)=\Gamma(T)$ requirement $x_{k+1} \approx x_{k}-v_{\text {group }}\left(\rho_{R}\right) T$, or

$$
\frac{\left|x_{k+1}-x_{k}\right|}{T} \approx\left(\frac{\partial j}{\partial \rho}\right)_{\rho=\rho_{R}}=\Delta .
$$

The distance between sawteeth converges monotonically and rapidly to the predicted value: indeed, as follows from Eq. (11), $\lim _{k \rightarrow \infty}\left|x_{k}-x_{0}\right| / T=\left(k+\frac{1}{2}-\frac{2 \lambda_{c}}{1-2 \lambda_{c}}\right)(\partial j / \partial \rho)_{\rho=\rho_{R}}$. Consequently, the sum of all deviations does not exceed $10 \%$ of the predicted limiting distance $\Delta$. Hence one can measure derivative of the flux $(\partial j / \partial \rho)_{\rho=\rho_{R}}$ directly by measuring the distance between the sawteeth. The estimate of $(\partial j / \partial \rho)_{\rho=\rho_{R}}$ from Eq. (31) for $k=1$ (the first and the best-visible sawtooth) induces relative error less than $4 \%$.

Finally, the amplitude of density variations $\delta \rho(x)$ can be estimated as $\left|h_{k}^{0}-g_{k}^{0}\right|$ at a distance $\left|x_{k}\right| \approx T k \Delta$. From Eqs. (9) and (10) we obtain 


$$
\delta \rho(-T k \Delta) \approx \frac{1-2 \lambda_{c}}{2 k}=O\left(\frac{1}{k}\right)
$$

In most of the paper, a semi-infinite system was considered. The analogy of the sawtooth structure with a shock and the picture of steady-state selection that emerges allows us to consider also finite systems with two open boundaries through which particles can enter or leave the system. We argue that the traffic light boundary condition represents a domain of the full phase diagram that includes part of the first-order transition between the low- and high-density phase. The maximal-current phase is reached only in the point where it meets the end of the first order transition line.

\section{ACKNOWLEDGMENTS}

V.P. thanks the IFF, Forschungszentrum Jülich, where a part of the work was completed, for the hospitality and the University of Salerno for providing a two year research grant during which this work was performed. M.S. acknowledges partial support from a MUR-PRIN-2005 initiative Transport properties of classical and quantum systems. G.M.S. thanks the Department of Physics "E.R. Caianiello" for partial support and for the kind hospitality received during the initial stages of this work.
[1] B. Schmittmann and R. K. P. Zia, in Phase Transitions and Critical Phenomena, edited by C. Domb and J. L. Lebowitz (Academic, London, 1995), Vol. 15.

[2] V. Privman, Nonequilibrium Statistical Mechanics in One Dimension (Cambridge University Press, Cambridge, 1997).

[3] G. M. Schütz, in Phase Transitions and Critical Phenomena, edited by C. Domb and J. L. Lebowitz (Academic, London, 2001), Vol. 19

[4] J. Krug, Phys. Rev. Lett. 67, 1882 (1991).

[5] V. Popkov and G. M. Schütz, Europhys. Lett. 48, 257 (1999).

[6] J. T. MacDonald, J. H. Gibbs, and A. C. Pipkin, Biopolymers 6, 1 (1968).

[7] G. M. Schütz, Int. J. Mod. Phys. B 11, 197 (1997).

[8] A. Parmeggiani, T. Franosch, and E. Frey, Phys. Rev. Lett. 90, 086601 (2003).

[9] V. Popkov, A. Rákos, R. D. Willmann, A. B. Kolomeisky, and G. M. Schütz, Phys. Rev. E 67, 066117 (2003).

[10] K. Nishinari, Y. Okada, A. Schadschneider, and D. Chowdhury, Phys. Rev. Lett. 95, 118101 (2005).

[11] D. Chowdhury, L. Santen, and A. Schadschneider, Phys. Rep. 329, 199 (2000).

[12] D. Helbing, Rev. Mod. Phys. 73, 1067 (2001).

[13] G. Schütz and E. Domany, J. Stat. Phys. 72, 277 (1993).

[14] B. Derrida, M. R. Evans, V. Hakim, and V. Pasquier, J. Phys. A 26, 1493 (1993).
[15] G. L. Eyink, J. L. Lebowitz, and H. Spohn, Commun. Math. Phys. 132, 253 (1990).

[16] C. Kipnis and C. Landim, Scaling Limits of Interacting Particle Systems (Springer, Berlin, 1999).

[17] C. Bahadoran, e-print arXiv:math.PR/0612094.

[18] G. B. Whitham, Linear and Nonlinear Waves (Pure and Applied Mathematics) (Wiley-Interscience, New York, 1974).

[19] V. Popkov and G. M. Schütz, J. Stat. Mech.: Theor. Exp. (2004) P12004.

[20] E. Brockfeld, R. Barlovic, A. Schadschneider, and M Schreckenberg, Phys. Rev. E 64, 056132 (2001).

[21] A. B. Kolomeisky, G. M. Schütz, E. B. Kolomeisky, and J. P. Straley, J. Phys. A 31, 6911 (1998).

[22] G. L. Eyink, J. L. Lebowitz, and H. Spohn, Commun. Math. Phys. 140, 119 (1991).

[23] F. Calogero and S. De Lillo, J. Math. Phys. 32, 99 (1991).

[24] A. Rakos, M. Paessens, and G. M. Schütz, Phys. Rev. Lett. 91, 238302 (2003).

[25] M. R. Evans, R. Juhasz, and L. Santen, Phys. Rev. E 68, 026117 (2003).

[26] S. B. Dutta and M. Barma, Phys. Rev. E 67, 061111 (2003).

[27] For a driven particle model with arbitrary current-density relation $j(\rho)$ which has a single maximum $\max j(\rho)=j\left(\rho^{*}\right)=j_{\max }$, it is expected that the stationary flux through the boundary equals the maximal possible flux $j_{\text {max }}$, i.e., $\lim _{\nu \rightarrow \infty} \rho_{R}^{\text {eff }}(\nu)=\rho^{*}$. 